\title{
Relationship between voluntary control of alpha activity level through auditory feedback and degree of eye convergence
}

\author{
ROBERT G. EASON and ROBERTA SADLER \\ University of North Carolina, Greensboro, North Carolina 27412
}

\begin{abstract}
Various studies have implicated the oculomotor system as possibly being involved in the biofeedback control of alpha activity with the eyes closed. This study investigated the effect of degree of eye convergence (and accompanying changes in accommodation) on alpha activity level both with the eyes open and closed. The effectiveness of eye convergence as a means of voluntarily controlling alpha activity level with the aid of EEG- or EOG-modulated auditory feedback also was investigated. Degree of eye convergence was found to be inversely related to alpha level both with the eyes open and closed. The two variables also were found to covary when subjects attempted, with the aid of biofeedback, to generate high or low levels of alpha activity with their eyes closed. The activation of visual cortex as manifested through changes in alpha activity appears to be increased when the eyes assume a position for viewing near objects, even when they are closed.
\end{abstract}

Since Kamiya's demonstration $(1968,1969)$ that, with appropriate feedback, one's alpha activity level can be brought under some degree of voluntary control, little objective evidence has been forthcoming on the nature of the mediational mechanisms responsible for such control. In view of the known importance of activational and attentional factors in the modulation of EEG activity (Lindsley, 1960), it is often assumed that such factors are internally manipulated by an individual when voluntarily altering this alpha activity level. Although direct evidence in support of this assumption is limited, at least one study has provided some psychophysiological data in support of it (Eason \& Sadler, Note 1; Sadler \& Eason, Note 2).

Although self-induced changes in activation level may be involved, a number of studies investigating the influence of visual factors on the EEG strongly implicate the oculomotor system as being a potentially important mediator in voluntary alpha control. With the eyes open, EEG activity has been shown to be influenced by variations in fixation and accommodation (Adrian \& Matthews, 1934; Oswald, 1959) as well as by complex tracking movements (Mulholland \& Peper, 1971). Also, stimulus input variables related to the clarity, texture, and intensity of the visual field have been shown to have a marked influence (Chapman, Shelburne, \& Bragdon, 1970; Lehman, Beeler, \& Fender, 1965; Lehtonen \& Lehtinen, 1972; Lynch, Paskewitz, \& Orne, 1974; Paskewitz \& Orne, 1973).

Mulholland and Evans $(1965,1966)$ observed that even when the eyes are closed, moving them to an elevated position results in an enhancement of alpha activity. Dewan (1967) then observed that the apparent relation-

This study was presented orally at the Psychonomic Society meetings held in Denver in 1975. ship between alpha enhancement and elevation of the closed eyes could be abolished by convergence of the elevated eyes. He concluded that alpha enhancement was not due to eye elevation per se but rather to a normal tendency for the eyes to diverge and for the accommodation system to become more relaxed as the eyes are rotated upward. Conversely, a downward movement of the closed eyes is accompanied by increased convergence and accommodation for near vision, leading to alpha suppression. In accord with Dewan's hypothesis, Chapman et al. (1970) observed that the enhancing effects of upward shifts of the eyes on alpha level did not occur when accommodation and degree of convergence were held constant.

In view of the above observations, the present study was undertaken to test the hypothesis that the relatively relaxed lens accommodation and eye divergence which accompanies far vision is associated with relatively high levels of alpha activity, whereas the more pronounced convergence and accommodation appropriate for near vision is associated with relatively low levels. A second purpose was to ascertain whether degree of eye convergence may constitute an effective strategy for voluntarily controlling alpha level in a feedback situation with the eyes closed.

\section{METHOD}

\section{Subjects and Experimental Design}

Ten students, three graduate and seven undergraduate, served as subjects. One of them (R.S.) had had considerable practice controlling alpha through participation in an earlier biofeedback experiment; the others had had no prior experience.

Each subject participated in a single experimental session approximately $1 \mathrm{~h}$ in duration. A session consisted of six 1-min trials. Thus, there were a total of $361-\mathrm{min}$ trials per subject.

In the first two blocks the subjects, with eyes closed, attempted to maintain high and low alpha levels on alternate 
trials while receiving EEG-modulated auditory feedback. These trials provided practice at alpha control preliminary to the introduction of the eye vergence manipulation.

In the next two blocks ( 3 and 4 ), the subjects kept their eyes open. On alternate trials they fixated a point located $1 \mathrm{~m}$ away (far condition) or $10 \mathrm{~cm}$ away (near condition). During Block 3 the subjects received only visual feedback; during Block 4 they received eye vergence controlled auditory feedback in addition to visual feedback. The angular difference between the near and far fixation points was approximately $20 \mathrm{deg}$.

In the last two blocks of trials, the subjects were again required to keep their eyes closed. In Block 5 they attempted on alternate trials to keep their eyes either converged or diverged with the aid of eye vergence controlled auditory feedback. On Block 6 they attempted, as in the first two trial blocks, to maintain high and low levels of alpha on alternate trials with the aid of EEG-modulated auditory feedback.

\section{Apparatus and Procedure}

Subjects sat in an electrically shielded, semidarkened room during the recording session. A Grass Model 7 polygraph, with appropriate preamplifiers, recorded EEG activity and the electrooculogram (EOG). EEG activity was recorded with an active electrode placed at position $\mathrm{O}_{\mathrm{z}}$ (about $2.5 \mathrm{~cm}$ above the inion along the midline of the occiput) and with a reference electrode at tached to the right earlobe. Unfiltered EEG activity was recorded in unintegrated form. The filtered alpha band component $(8-12 \mathrm{~Hz})$ was recorded in integrated form. Alpha level feedback was provided continuously to the subject through the pitch of a tone, by imposing the integrated alpha signal on the input of a frequency modulated tone generator, the output of which was connected to a speaker located in the ceiling of the subject's room.

Vergence eye movements were recorded electrooculographically. The EOG was obtained with electrodes located behind the external canthi of the eyes and on the bridge of the nose. The nose electrode was connected to one side of the dc preamplifier input; the external canthi electrodes were yoked and connected to the other side. This electrode configuration was quite sensitive to vergence movements, while being relatively insensitive to conjugate movements. Gold cup electrodes filled with Grass electrode cream containing no chlorides permitted dc recording with relatively low drift rates.

At the beginning and end of each trial, the subject was instructed to fixate the far target. This fixation provided the anchor point for determining the degree of convergence during a given trial and for determining the amount of drift during the course of the trial due to electrode polarization.

Before beginning the recording session for a given subject, it was determined that both the near and far fixation points were clearly visible to him in the dimly lit room. At the beginning of each block of trials, appropriate instructions were given to the subjects as to the nature of the task. White noise was used to mask extraneous sounds during each recording period.

\section{RESULTS}

\section{Quantification of Polygraph Records}

A sample recording of the unintegrated EEG, integrated and filtered $(8-12 \mathrm{~Hz})$ alpha activity, and the EOG is shown in Figure 1 to illustrate the quantification procedures. The record on the left is a 1 -min segment obtained while the subject attempted to maintain a high alpha level with eyes closed while receiving alphamodulated tone feedback. The middle segment shows the subject resting with eyes open. In the 1-min segment on the right, the subject attempted to keep alpha level as low as possible with eyes closed and with the aid of alpha-modulated tone feedback.

As depicted in Figure 1, both alpha level and degree

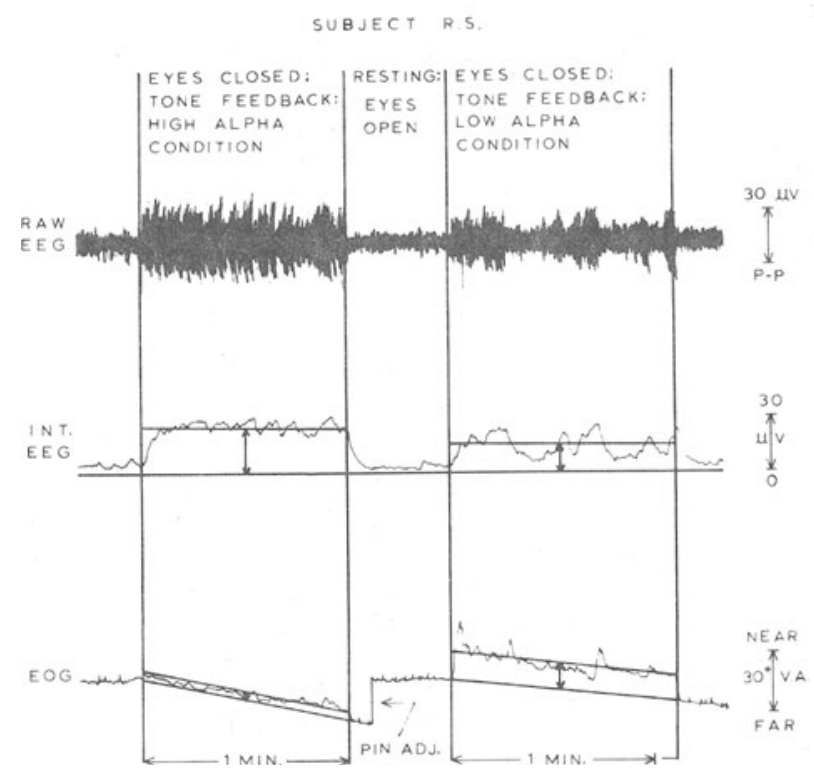

Figure 1. Sample polygraph record of raw EEG (top row), integrated filtered alpha activity (middle row), and the EOG (bottom row).

of convergence per 1-min interval were quantified for each subject by drawing a visually "best fit" line through the integrated alpha and EOG records parallel to their respective baselines, and expressing the perpendicular distance between the two parallel lines either in microvolts (for alpha activity) or visual angle (for the EOG). The "best fit" lines were visual averages which could be drawn with a very high degree of reliability by the same, as well as by two different scorers. Lines drawn independently through the same data segments by the two scorers frequently superimposed, and virtually never differed in the vertical dimension by more than $1 \mathrm{~mm}$.

While the Grass equipment provided a baseline for the integrated alpha activity, it was necessary to provide a standard reference point for establishing an EOG "baseline." This was accomplished by instructing the subject to fixate the far target immediately before and after each 1-min trial. A straight line connecting the two segments of the EOG recorded during these pre- and posttrial fixations constituted the standardized reference, or "baseline," for determining the degree of eye convergence during a given trial.

\section{Group Findings}

The results obtained for the last three trial blocks of the session, based on the group data, are depicted in Figure 2, wherein the means for alpha level and degree of eye convergence are plotted. Data obtained in the first two trial blocks are not shown, since these constituted practice trials for all subjects except one and no group relationships between alpha level and degreè of eye convergence were either expected or observed. The results obtained in the third trial block, also not shown, were essentially a duplicate of those shown for the fourth block in Figure 2 (left-hand graph).

General inspection of the figure reveals that increased 
MEANS (GROUP DATA)

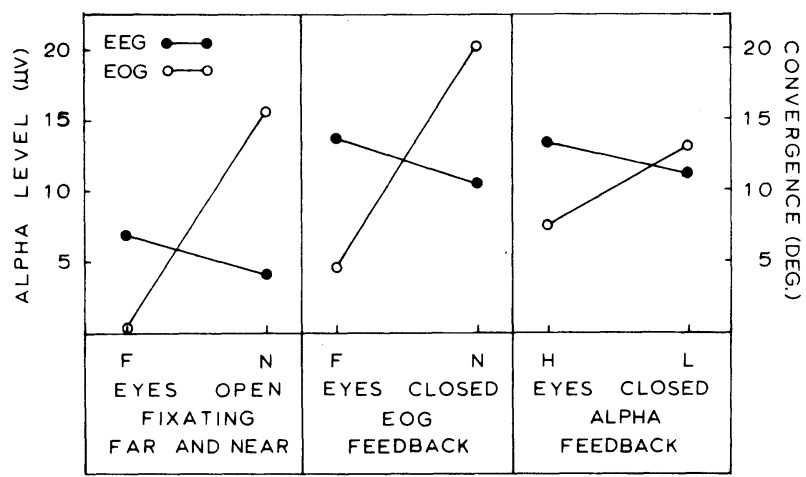

Figure 2. Changes in group means of integrated alpha activity (EEG) and EOG as a function of (1) near and far fixation (left graph), (2) degree of eye convergence with eyes closed (middle graph), and (3) alpha-modulated tone feedback with eyes closed (right graph).

convergence was generally accompanied by decreased alpha level and vice versa. When the subjects fixated the near or far targets while receiving EOG-modulated tone feedback (left-hand graph), alpha level was found to be significantly lower for the near condition $(p<.01)$. Not surprisingly, the change in the EOG was also significant $(p<.001)$, since the subjects had no difficulty maintaining fixation on the near and far targets with their eyes open.

Even with their eyes closed, the subjects successfully maintained prolonged convergence or divergence, roughly comparable to that which would have been required to fixate the near or far target, through the aid of EOGmodulated tone feedback (center graph). The average difference in eye convergence between the two vergence conditions was $15.7 \mathrm{deg}$; this difference was significant at the .001 level of confidence. Of greater interest is the fact that a statistically significant difference $(\mathrm{p}<.05)$ in the amount of alpha activity also was obtained as a function of degree of convergence, less activity having occurred during the high convergence condition.

When the subjects attempted to maintain either high or low alpha levels with their eyes closed through the aid of alpha-modulated tone feedback (right-hand graph), the difference in alpha level for the high and low conditions was significant $(p<.05)$ even though 9 of the 10 subjects had had no prior practice at voluntary alpha control other than the first two trial blocks in the present experiment. Greater eye convergence also was observed, on the average, when the subjects tried to maintain a low alpha activity level. However, the difference in vergence between the high and low alpha conditions only approached significance at the $5 \%$ level of confidence $(p<.10)$.

A comparison of the alpha activity data for the EOGand alpha-modulated feedback conditions (center and right-hand graphs) suggests the subjects were just as successful at "controlling" their alpha activity levels with eyes closed through EOG feedback as they were through more direct alpha feedback.

\section{Experienced Subject's Data}

The results summarized above for the group as a whole were markedly accentuated in the polygraphic tracings of the one experienced subject (R.S.). Her data are presented in their entirety in Figure 3 for all experimental conditions to demonstrate the magnitude and consistency with which the eye vergence/alpha activity relationship manifested itself.

The top two rows show superimposed tracings of integrated alpha (top row) and EOG (second row) obtained during each 1 -min period of the first two trial blocks. Visual inspection of these tracings reveals that the eyes were less converged during the high than during the low alpha condition, and that there was far more vergence eye movement activity and overall variability in the EOG during the low condition. Correspondingly, there was more variability in the integrated alpha records during the low than during the high alpha condition.

The middle two rows of tracings show integrated alpha activity level and vergence movements when the subject, with eyes open, fixated the far and near targets (Trial Blocks 3 and 4). It is readily apparent that the
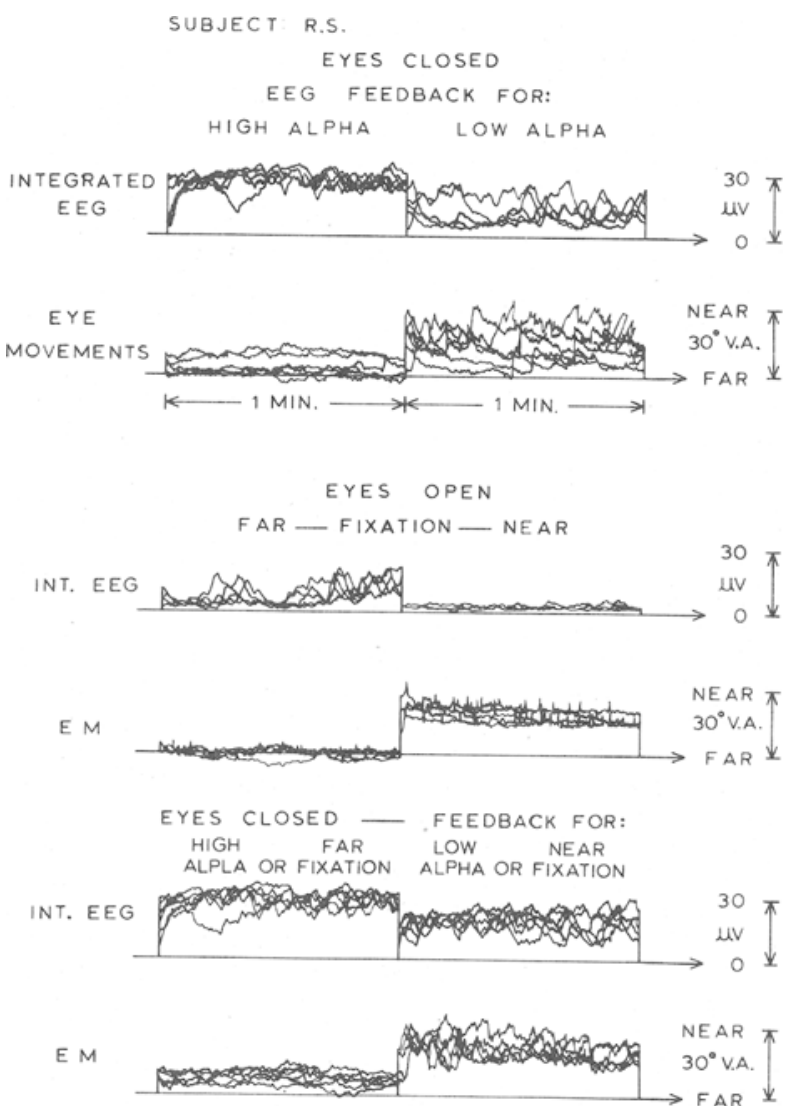

Figure 3. Polygraphic tracings obtained from a subject highly skilled at controlling alpha activity with the aid of auditory feedback. Rows 1, 3, and 5 are integrated alpha tracings; rows 2,4 , and 6 are vergence eye movement tracings. See text for further explanation. 
alpha activity was of greater magnitude and more variable during far fixation. During near fixation, alpha activity was reduced to a highly stable, low amplitude level characteristic of desynchronized EEG activity.

Although EOG-modulated feedback was given to the subject during Block 5 and alpha-modulated feedback during Block 6, the tracings obtained during the two blocks have been superimposed (rows 5 and 6) to illustrate the highly consistent covariation which occurred between integrated alpha activity and the EOG regardless of the type of feedback used. Visual inspection of the tracings reveals that alpha activity was influenced just as markedly by eye convergence or divergence through EOG-modulated tone feedback for this subject, with her eyes closed, as by instructions to keep alpha level as low or high as possible with the aid of alpha-modulated tone feedback.

Although the data were less consistent for the inexperienced subjects, similar results were manifested in their polygraph records during the last three trial blocks, as the group statistical analyses reported above imply.

\section{DISCUSSION}

The above results provide evidence that degree of eye convergence is linked to alpha activity level, convergence being associated with relatively low levels of alpha activity and divergence with relatively high levels. Such linkage might be due to neural feedback to those areas of the brain responsible for the modulation of alpha activity which arises from (1) the musculature involved in vergence movements, (2) intraocular musculature controlling accommodation, or (3) both. It seems probable that both afferent inputs may be involved, since convergence and accommodation mechanisms are known to be neruologically linked (Davson, 1972, pp. 409-413).

With regard to voluntary alpha control when the eyes are closed, the above findings indicate that one effective strategy, if not a frequently used one, would be simply to vary degree of eye convergence. While variations in vertical eye position have been shown to affect alpha activity (Mulholland \& Evans, 1965,1966 ), our data along with the earlier findings of Chapman et al. (1970), provide further indirect support for Dewan's (1967) view that the enhancing and suppressing effects may not be due to the elevation and lowering of the eyes per se but rather to variations in degree of eye convergence correlated with such vertical displacement.

When attempting to control alpha level through alphamodulated tone feedback, subjects probably test a number of strategies and finally settle on one or two which seem to work best for them. Since both attentional and eye vergence mechanisms seem to be involved in the modulation of alpha activity, perhaps one of the more effective strategies for manipulating alpha activity level through feedback with eyes closed would be one which utilizes both types of mechanisms. It seems probable that certain kinds of visual imagery or cognitions not only may affect the general activity level of the cortex directly but also may serve to induce vergence and lens accommodation changes which further alter the activity level of the visual cortex.

Since there is no kinesthetic feedback to the eye muscles (Brindley \& Merton, 1960), it is entirely possible that some individuals highly skilled at controlling their alpha activity levels utilize strategies which induce changes in eye vergence and accommodation without realizing it.

\section{REFERENCE NOTES}

1. Eason, R. G., \& Sadler, R. R. Voluntary alpha control, visually evoked potentials, and peripheral physiological indicants of activation. Manuscript submitted for publication, 1976.

2. Sadler, R. R., \& Eason, R. G. Physiological correlates of voluntary production and suppression of alpha activity. Paper presented at the meeting of the Southeastern Psychological Association, Hollywood, Florida, April 1975.

\section{REFERENCES}

Adrian, E. D., \& Matthews, B. C. H. The Berger rhythm: Potential alterations from the occipital lobes in man. Brain, 1934, 57, 355-385.

Brindley, G., \& Merton, P. The absence of position sense in the human eye. Journal of Physiology, 1960, 153, 127-130.

Chapman, R. M., Shelburne, S. A., \& Bragdon, H. R. EEG alpha activity influenced by visual input and not by eye position. Electroencephalography and Clinical Neurophysiology, 1970, 28, 183-189.

Davson, H. The physiology of the eye. New York: Academic Press, 1972. Pp. 409-413.

Dewan, E. M. Occipital alpha rhythm, eye position and lens accommodation. Nature, 1967, 214, 975-977.

KAMTYA, J. Conscious control of brain waves. Psychology Today, 1968, 1, 57-60.

KAMIYA, J. Operant control of EEG alpha rhythm and some of its reported effects on consciousness. In C. Tart (Ed.), Altered states of consciousness. New York: Wiley, 1969.

Lehmann, D., Beeler, G. W., \& Fender, D. H. Changes in patterns of the human electroencephalogram during fluctuations of perception of stabilized retinal images. Electroencephalography and Clinical Neurophysiology, 1965, 19, 336-343.

Lehtonen, J. B., \& Lehtinen, J. Alpha thythm and uniform visual field in man. Electroencephalography and Clinical Neurophysiology, 1972, 32, 139-147.

LiNDsLey, D. B. Attention, consciousness, sleep and wakefulness. In J. Field (Ed.), Handbook of physiology: Neurophysiology (Vol. 3). Washington, D.C: American Physiological Society, 1960.

Lynch, J. J., PAskewitz, D. A., \& ORne, M. T. Some factors , in the feedback control of human alpha rhythm. Psychosomatic Medicine, 1974, 36, 399-411.

Mulholland, T., \& Evans, C. R. An unexpected artefact in the human electroencephalogram concerning the alpha rhythm and the orientation of the eyes. Nature, 1965, 207, 36-37.

Mulholland, T., \& Evans, C. R. Oculomotor function and the alpha activation cycle. Nature, 1966, 211, 1278-1279.

Mulholland, T. B., \& Peper, E. Occipital alpha and accommodative vergence, pursuit tracking, and fast eye movements. Psychophysiology, 1971, 8, 556-575.

Oswald, I. The human alpha rhythm and visual alertness. Electroencephalography and Clinical Neurophysiology, 1959, 11, 601. (Abstract)

Paskewitz, D. A., \& OrNe, M. T. Visual effects on alpha feedback training. Science, 1973, 181, 360-363.

(Received for publication August 19, 1976.) 